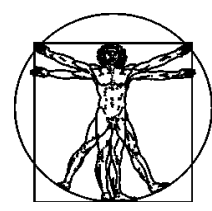

\title{
ИССЛЕДОВАНИЕ ЗАВИСИМОСТИ МЕЖДУ ДАВЛЕНИЕМ ВЫДЫХАЕМОГО ВОЗДУХА И ВНУТРИБРЮШНЫМ ДАВЛЕНИЕМ ЧЕЛОВЕКА
}

\author{
В.С. Туктамышев ${ }^{1}$, Е.Ю. Касатова ${ }^{2}$, Ю.И. Няшин ${ }^{1}$ \\ ${ }^{1}$ Кафедра теоретической механики и биомеханики Пермского национального исследовательского \\ политехнического университета, Россия, 614990, Пермь, Комсомольский проспект, 29, e-mail: \\ helpinvader@list.ru \\ 2 Кафедра акушерства и гинекологии Пермского государственного медицинского университета имени \\ академика Е.А. Вагнера, Россия, 614000, Пермь, ул. Петропавловская, 26, e-mail: \\ elena.kasatova@googlemail.com
}

\begin{abstract}
Аннотация. Существующие на сегодняшний день методы измерения внутрибрюшного давления являются инвазивными и, следовательно, сложными в реализации. Поэтому разработка доступных неинвазивных способов позволила бы лучше изучить явления, связанные с повышением давления в полости живота человека. В представленной работе описывается экспериментальное исследование взаимовлияния давления выдыхаемого воздуха и внутрибрюшного давления. Наличие зависимости между этими величинами основывается на предположении о том, что давление брюшных масс в положении лежа на спине одинаково воздействует на все поверхности, располагающиеся в полости живота, в частности на диафрагму и стенку мочевого пузыря. Измерение давления в мочевом пузыре считается эталонным методом определения внутрибрюшного давления. Таким образом, установление связи между внутрипузырным давлением и давлением выдыхаемого воздуха, определение которого может полностью осуществляться вне организма человека, позволит приблизиться к решению вопроса о создании неинвазивной процедуры измерения внутрибрюшного давления. Результаты эксперимента подтвердили наличие зависимости между двумя исследуемыми величинами. Показано, что для отдельно взятого человека данная зависимость близка к линейной. Тем не менее в общем случае наблюдается достаточно сильное расхождение между значениями измеряемых давлений. По всей вероятности, это связано с тем, что в процессе выдоха важную роль играют и другие факторы, такие как натяжение дыхательных мышц, механические свойства тканей легких, возможность самопроизвольного регулирования интенсивности выдоха. В связи с этим детальное исследование взаимозависимости давления выдыхаемого воздуха и внутрибрюшного давления требует проведения новых экспериментов, учитывающих влияние дополнительных параметров.
\end{abstract}

Ключевые слова: внутрибрюшное давление, давление выдыхаемого воздуха, методы измерения внутрибрюшного давления.

\section{ВВЕДЕНИЕ}

Внутрибрюшное давление обусловлено взаимной компрессией внутрибрюшных масс и их давлением на оболочку брюшной полости [3]. Влияние повышенного внутрибрюшного давления на деятельность сердечно-сосудистой, мочевыделительной и дыхательной систем описано в многочисленных научных обзорах $[8,15,18]$. Кроме

(С) Туктамышев В.С., Касатова Е.Ю., Няшин Ю.И., 2015

Туктамышев Вадим Саитзянович, к.ф.-м.н., доцент кафедры теоретической механики и биомеханики, Пермь

Касатова Елена Юрьевна, к.м.н., доцент кафедры акушерства и гинекологии, Пермь

Няшин Юрий Иванович, д.т.н., профессор кафедры теоретической механики и биомеханики, Пермь 
того, давление в полости живота играет важную роль в снижении уровня механических напряжений, возникающих в повседневной жизни в межпозвоночных дисках поясничного отдела позвоночника [2, 4-7, 10-12]. Опасные для здоровья и жизни человека величины внутрибрюшного давления определяются принятыми стандартами [16]. При этом внутрибрюшным считается давление жидкости внутри мочевого пузыря.

На сегодняшний день известны различные способы измерения внутрибрюшного давления. Наиболее точным из них является прямой метод, предложенный в 1931 г. Оверхольтом (R. Overholt) [17]. Суть этого метода заключается в том, что измерение производится с помощью катетера, установленного непосредственно в полость живота. Несмотря на свою точность, такой способ имеет существенный недостаток, связанный с необходимостью хирургических разрезов стенки брюшной полости. Поэтому более широкое распространение получили непрямые методы, использующие полые органы, расположенные в полости живота или граничащие с ней. К таковым относятся желудок, прямая кишка, нижняя полая вена и мочевой пузырь [9]. Для измерения в какой-либо из этих органов (кроме мочевого пузыря) устанавливается манометрический датчик. Давление, регистрируемое таким образом внутри соответствующего органа, оказывается достаточно точным по отношению к внутрибрюшному давлению, измеренному прямым способом. Тем не менее такая методика является довольно сложной по исполнению и требует специальной аппаратуры.

Поэтому в настоящее время наиболее часто используется трансвезикальный (через мочевой пузырь) метод измерения внутрибрюшного давления, который в 1984 г. предложил хирург I. Kron [14]. Этот метод основан на введении гибкого катетера (катетера Фолея) в мочевой пузырь через мочеиспускательный канал. В положении пациента лежа на спине по катетеру вводится некоторое количество физиологического раствора. После этого свободный конец катетера соединяется с мерной трубкой, по высоте столбика жидкости в которой и определяется внутрипузырное давление. Стенка мочевого пузыря при этом выполняет роль мембраны, которая с большой степенью точности передает давление в полости живота [13]. Таким образом, за величину внутрибрюшного давления можно принимать измеряемое описанным способом значение давления внутри мочевого пузыря. По сравнению с остальными методами измерения внутрибрюшного давления трансвезикальный метод является наиболее предпочтительным с точки зрения безопасности, точности и простоты исполнения.

Процедура измерения внутрибрюшного давления через мочевой пузырь главным образом используется в отделениях интенсивной терапии и реанимации, так как, несмотря на перечисленные достоинства, данная методика инвазивна. Ее применение в многоцентровых научных исследованиях, а также при профилактике внутрибрюшной гипертензии ограничено. Поэтому разработка более доступных неинвазивных методов прежде всего позволила бы шире взглянуть на эффекты, связанные с повышением давления в полости живота.

В представленной работе с помощью серии экспериментов сделан первый шаг к установлению связи между давлением выдыхаемого воздуха и внутрибрюшным давлением, измеряемым через мочевой пузырь. При этом исследуется пассивный выдох, т.е. выдох, который не сопровождается активностью мышц, участвующих в механизме дыхания. Предположение о взаимозависимости этих величин основано на гипотезе о том, что в положении лежа на спине внутрибрюшное давление в равной степени воздействует на все поверхности в полости живота, в частности на диафрагму и стенку мочевого пузыря [9]. Регистрация давления выдыхаемого воздуха может проводиться с помощью устройств, элементы которых не требуют расположения внутри организма человека, что при установлении искомой взаимосвязи позволит упростить процедуру измерения внутрибрюшного давления. 


\section{МАТЕРИАЛЫ И МЕТОДЫ}

В эксперименте приняли участие четыре женщины в возрасте от 22 до 42 лет

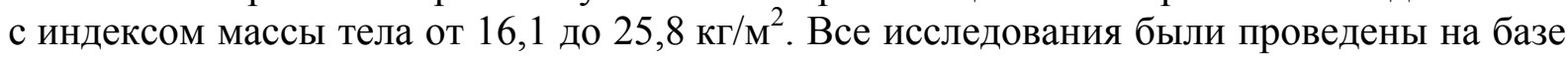
кафедры акушерства и гинекологии Пермского государственного медицинского университета имени академика Е.А. Вагнера.

Схема эксперимента показана на рис. 1. Для измерения внутрипузырного давления была использована специальная стерильная закрытая система «UnoMeter ${ }^{T M}$ Abdo-Pressure ${ }^{T M} »$ (Unomedical, Дания), являющаяся на сегодняшний день наиболее предпочтительной среди устройств, измеряющих внутрибрюшное давление [19]. Регистрация объемов вдыхаемого воздуха производилась с помощью компьютерного спирометра «Спиро-Спектр» (Нейрософт, Россия) [1]. Давление выдыхаемого воздуха с достаточной степенью точности определялось жидкостным $U$-образным манометром, соединяемым посредством трубок с герметичной маской.

Ход эксперимента можно представить в виде следующих шагов:

1) на лицо испытуемого накладывается плотно прилегающая маска, которая имеет клапан для дыхания и отверстия для подсоединения трубок, соединяющих маску со спирометром и манометром;

2) испытуемый вдыхает определенное количество воздуха, объем которого измеряется с помощью спирометра, после чего клапан для дыхания на маске закрывается;

3) производится пассивный выдох, при этом выдыхаемый воздух попадает в замкнутый контур, образуемый воздухоносными путями испытуемого, герметичной маской, трубками и манометром;

4) давление воздуха в контуре фиксируется с помощью показаний манометра, одновременно с этим по шкале мерной трубки прибора «UnoMeter ${ }^{T M}$ Abdo-Pressure $^{T M}$ » определяется внутрибрюшное давление.

Объемы воздуха при вдохе, для которых были проведены исследования соответствий между давлением выдыхаемого воздуха и внутрибрюшным давлением, составляли 0,$5 ; 1 ; 1,5$ литра.

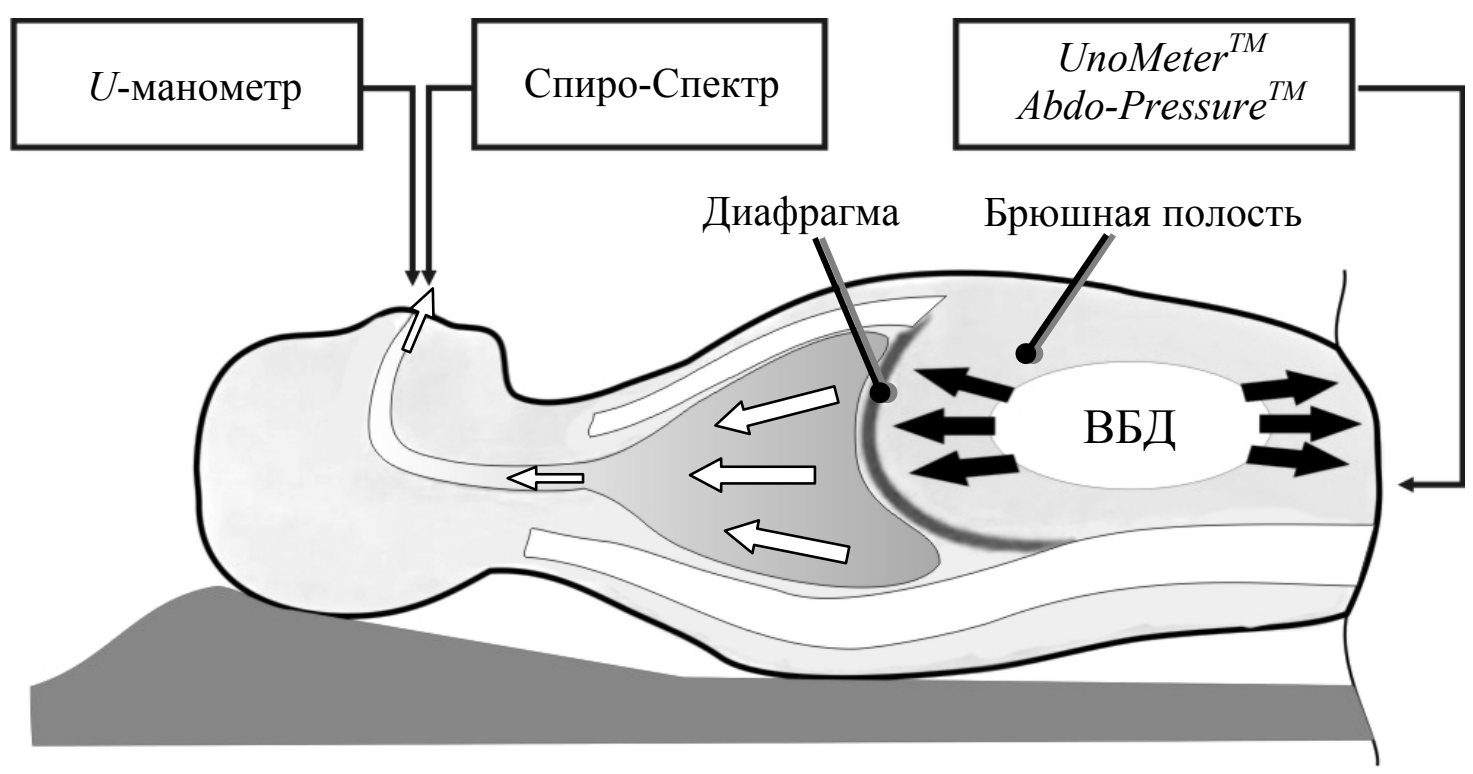

Рис. 1. Схема эксперимента. $U$-манометр - $U$-образный манометр; Спиро-Спектр компьютерный спирометр; UnoMeter ${ }^{T M}$ Abdo-Pressure ${ }^{T M}-$ система для измерения внутрибрюшного давления, ВБД - внутрибрюшное давление 


\section{РЕЗУЛЬТАТЫ И ОБСУЖДЕНИЕ}

Результаты исследования приведены в таблице. Для наглядности на рис. 2 представлено сопоставление полученных значений внутрибрюшного давления $P_{1}$ и давления выдыхаемого воздуха $P_{2}$.

Результаты исследования

\begin{tabular}{|c|c|c|c|}
\hline Испытуемый & $\begin{array}{c}\text { Внутрибрюшное } \\
\text { давление, мм рт. ст. }\end{array}$ & $\begin{array}{c}\text { Давление выдыхаемого } \\
\text { воздуха, мм рт. ст. }\end{array}$ & Объем вдоха, л \\
\hline \multirow{3}{*}{ № 1} & 5 & 8,8 & 1,5 \\
\hline & 3,5 & 7 & 1 \\
\hline & 2,8 & 5,5 & 0,5 \\
\hline \multirow{3}{*}{ № 2} & 15 & 17,7 & 1,5 \\
\hline & 13 & 14,7 & 1 \\
\hline & 9 & 12,5 & 0,5 \\
\hline \multirow{3}{*}{ № 3} & 6,5 & 10,7 & 1,5 \\
\hline & 5,5 & 7,4 & 1 \\
\hline & 5 & 4 & 0,5 \\
\hline \multirow{3}{*}{ № 4} & 14 & 9,6 & 1,5 \\
\hline & 11 & 7,4 & 1 \\
\hline & 10 & 6,3 & 0,5 \\
\hline
\end{tabular}

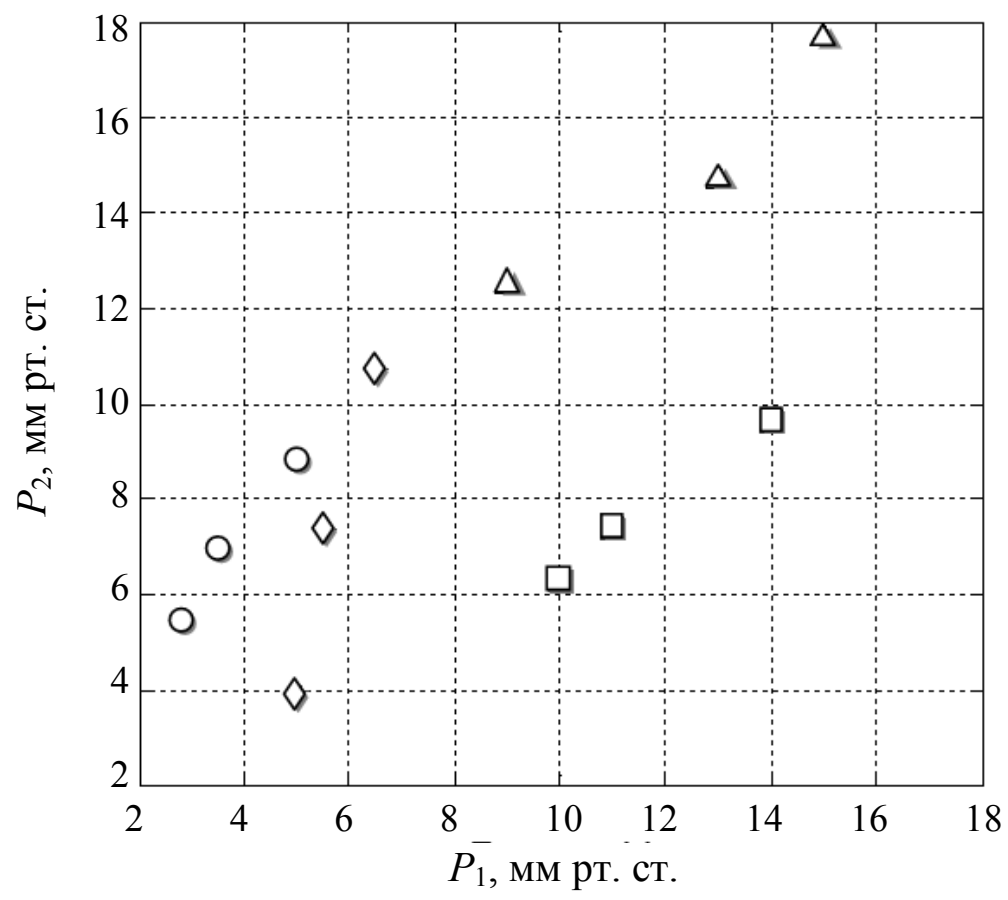

Рис. 2. Сопоставление измеренных значений давлений $P_{1}$ и $P_{2}$ : ○ - испытуемый № $1 ; \Delta$ - испытуемый № 2; $\diamond$ - испытуемый № 3 ; $\square-$ испытуемый № 4 
Из приведенных выше данных видно, что давление выдыхаемого воздуха и давление в полости живота, измеренное с помощью трансвезикального метода, сопоставляются качественно, т.е. рост $P_{1}$ определяет повышение $P_{2}$. При этом точки, соответствующие трем последовательным измерениям для каждого из испытуемых, практически лежат на одной прямой (см. рис. 2). Таким образом, исследование показывает, что для отдельно взятого человека зависимость давления выдыхаемого воздуха от внутрибрюшного давления с большой долей вероятности является линейной. Однако ни одна из таких линейных закономерностей не распространяется на общий случай. Кроме того, наблюдается достаточно сильное расхождение между значениями давлений $P_{1}$ и $P_{2}$. Это связано, по-видимому, с тем, что давление выдыхаемого воздуха определяется не только давлением брюшных масс на диафрагму. В процессе выдоха немаловажными факторами оказываются натяжение дыхательных мышц, механические свойства тканей легких, возможность самопроизвольного регулирования интенсивности выдоха.

Таким образом, для более детального исследования взаимозависимости давления выдыхаемого воздуха и внутрибрюшного давления требуется проведение целого ряда экспериментов, которые учитывали бы дополнительные параметры. К этим параметрам можно отнести, например, дыхательные объемы при спокойном и форсированном дыхании, возможную активность дыхательных мышц в момент измерения давления выдыхаемого воздуха и др.

\section{ЗАКЛЮЧЕНИЕ}

Представленный в данной работе обзор позволяет сделать вывод о важности внутрибрюшного давления. Вместе с другими показателями состояния организма человека данный параметр может использоваться для комплексного анализа различных патологий. Длительное повышение внутрибрюшного давления (внутрибрюшная гипертензия) проявляется в виде системных нарушений и может привести к тяжелым последствиям. Таким образом, изучение внутрибрюшной гипертензии человека является актуальной задачей. При этом важным направлением в контексте данной задачи является биомеханическое моделирование, которое, по мнению авторов, позволит ответить на некоторые вопросы о взаимодействии внутренних органов и сосудов брюшной полости.

\section{СПИСОК ЛИТЕРАТУРЫ}

1. Спиро-Спектр [Электронный ресурс]. - URL: http://www.neurosoft.ru/rus/product/spirospectrum/index.aspx (дата обращения: 4.03.2014).

2. Туктамышев В.С., Безматерных В.В. Моделирование влияния внутрибрюшного давления на нагружение позвоночного столба // Современные проблемы науки и образования. - 2014. - № 3. - С. 704.

3. Туктамышев В.С., Кучумов А.Г., Няшин Ю.И., Самарцев В.А., Касатова Е.Ю. Внутрибрюшное давление человека // Российский журнал биомеханики. - 2013. - Т. 17, № 1. - С. $22-31$.

4. Туктамышев В.С., Соломатина Н.В. Влияние внутрибрюшного давления на состояние поясничного отдела позвоночника // Фундаментальные исследования. - 2013. - № 8. - С. 77-81.

5. Bartelink D.L. The role of abdominal pressure in relieving the pressure on the lumbar intervertebral discs // Journal of Bone and Joint Surgery. - 1957. - Vol. 39. - P. 718-725.

6. Cholewicki J., Juluru K., McGill S.M. Intra-abdominal pressure mechanism for stabilizing the lumbar spine // Journal of Biomechanics - 1999. - Vol. 32. - P. 13-17.

7. Daggfeldt K., Thorstensson A. The role of intra-abdominal pressure in spinal unloading // Journal of Biomechanics. - 1997. - Vol. 30, № 11/12. - P. 1149-1155.

8. De Waele J.J., De Laet I., Malbrain M.L. Intra-abdominal hypertension and abdominal compartment syndrome: we have paid attention, now it is time to understand! // Acta Clinica Belgica. - 2007. Vol. 62. - S6-S8.

9. De Waele J.J., De Laet I., Malbrain M.L. Rational intra-abdominal pressure monitoring: how to do it? // Acta Clinica Belgica. - 2007. - Vol. 62. - P. 16-S25.

10. Essendrop M., Andersen T.B., Schibye B. Increase in spinal stability obtained at levels of intra-abdominal pressure and back muscle activity realistic to work situations // Applied Ergonomics. - 2002. - Vol. 33. P. 471-476. 
11. Hodges P.W., Cresswell A.G., Daggfeldt K., Thorstensson A. In vivo measurement of the effect of intraabdominal pressure on the lumbar spine // Journal of Biomechanics. - 2001. - Vol. 34. - P. 347-353.

12. Hodges P.W., Eriksson A.E., Shirley D., Gandevia S.C. Intra-abdominal pressure and abdominal wall muscular function: spinal unloading mechanism // Journal of Biomechanics. - 2005. - Vol. 38. - P. 18731880.

13. Joynt G.M., Ramsay S.J., Buckley T.A. Intra-abdominal hypertension - implications for the intensive care physician // Ann. Acad. Med. Singapore. - 2001. - Vol. 30, № 3. - P. 310-319.

14. Kron I.L., Harman P.K., Nolan S.P. The measurement of intra-abdominal pressure as a criterion for abdominal re-exploration // Annals of Surgery. - 1984. - Vol. 199, № 1. - P. 28-30.

15. Malbrain M.L. Abdominal pressure in the critically ill // Current Opinion in Critical Care. - 2000. - Vol. 6, № 1. - P. 17-29.

16. Malbrain M.L., Cheatham M.L., Kirkpatrick A., Sugrue M., Parr M., De Waele J., Balogh Z., Leppaniemi A., Olvera C., Ivatury R., D’Amours S., Wendon J., Hillman K., Johansson K., Kolkman K., Wilmer A. Results from the international conference of experts on intra-abdominal hypertension and abdominal compartment syndrome. I. Definitions // Intensive Care Medicine. - 2006. - Vol. 32, № 11. - P. 1722-1732.

17. Overholt R. Intraperitoneal pressure // Arch. Surg. - 1931. - Vol. 22. - P. 691-703.

18. Surgue M. Abdominal compartment syndrome // Current Opinion in Critical Care. - 2005. - Vol. 11, № 4, - P. 333-338.

19. UnoMeter ${ }^{\mathrm{TM}}$ Abdo-Pressure ${ }^{\mathrm{TM}}$ [Электронный pecypc]. - URL: http://www.convatec.co.uk/faecalmanagement/iap-monitoring/product-range/unometer-abdo-pressure (дата обращения: 4.03.2014).

\section{INVESTIGATION OF THE RELATIONSHIP BETWEEN THE EXPIRATION PRESSURE AND INTRA-ABDOMINAL PRESSURE OF HUMAN}

\section{V.S. Tuktamyshev, E.Y. Kasatova, Y.I. Nyashin (Perm, Russia)}

The existing methods for measuring intra-abdominal pressure are invasive and consequently difficult to implement. Therefore, the development of noninvasive methods available would allow us to better understand the phenomena associated with increased pressure in the abdomen of human. In the present paper, we describe an experimental study of expiration pressure and intra-abdominal pressure. Having a relationship between these variables is based on the assumption that the pressure of the abdominal masses in a supine position equally affects all surfaces, which are located in the abdominal cavity, in particular the diaphragm and the wall of the bladder. Measurement of pressure in the bladder is considered the reference method for determining the intra-abdominal pressure. Thus, the links between intravesical pressure and the pressure of expired air, the determination of which can be fully realized outside the human body, allow the approach to the issue of creating a noninvasive procedure for measuring intra-abdominal pressure. The experimental results have confirmed the relationship between the two variables under consideration. It is shown that for a single person, this relationship is close to linear. However, generally, there are large discrepancies between the values of measured pressures. In all likelihood, this is due to the fact that during expiration other factors are important, such as respiratory muscle tension, the mechanical properties of lung tissue, the possibility of regulating the intensity of spontaneous expiration. In this connection, a detailed study of the interdependence of the expiration pressure and intra-abdominal pressure requires new experiments taking into account the effect of additional parameters.

Key words: intra-abdominal pressure, expiration pressure, methods of measuring intraabdominal pressure, inspiratory volume. 